\title{
Effect of skylight configuration and sky type on the daylight impression of a room
}

\author{
P. Seuntiens, M. van Boven \& D. Sekulovski \\ Philips Research, Eindhoven, The Netherlands
}

\begin{abstract}
Today, daylight is considered to be the ultimate light source for performance lighting, ambiance creation, and healing environments. More and more architects design interior spaces using daylight not only to provide us with lighting to be able to see, but to connect us with the outside world and create beautiful daylight patterns inside. The light distribution in an interior space resulting from daylight is strongly connected with the architecture of the daylight opening and the sky type. Being able to understand the perceptual relation between architectural choices and the attractive qualities of daylight provides valuable insights for maximizing the daylight impression of a room.

In a visual perception experiment, we measured the effect of skylight configuration and sky type on the daylight impression in a room. This was done based on renderings of a room. A room with different skylight configurations (5x) was built in Sketch-up, and Indigo renderer was used to render the lighting effects in the room using HDR sky images (overcast, partly clouded with/without visible sun, clear sky). In a paired comparison set-up (70 comparisons), the participants had to indicate which picture (left or right) had the strongest daylight impression. The comparisons were shown to the participants in a randomized order. After the experiment, participants had to fill out a short questionnaire.

The statistical analysis showed a significant main effect of skylight configuration and sky type. In general, people preferred the larger rectangular skylights over the squared skylights in terms of daylight impression. The clear sky and partly clouded (sun visible) sky types resulted in a better daylight impression than the partly clouded (sun not visible). The daylight impression of an overcast sky was very low in general. The results of the questionnaire afterwards showed that the decision on daylight impression was mainly determined by 1) skylights (layout and view) and 2) daylight patterns (edge
\end{abstract}


sharpness and color temperature). No interaction effects between skylight configuration and sky type were found.

Keywords: daylight impression, skylight configuration, sky type, perception.

\section{Introduction}

Today, daylight is considered to be the ultimate light source for performance lighting, ambiance creation, and healing environments [1-4]. More and more architects design interior spaces using daylight not only to provide us with lighting to be able to see, but to connect us with the outside world and create beautiful daylight patterns inside. The light distribution in an interior space resulting from daylight is strongly connected with the architecture of the daylight opening and the sky type.

Daylight is the combination of direct sunlight and diffuse skylight. The atmospheric particles in the sky are responsible for scattering the shorter wavelengths of the sunlight spectrum, resulting in a blue appearance of the sky. The higher wavelengths are relatively unaffected resulting in warmer direct sunlight. Daylight inside buildings consists of four components in line with the CIE standards for the daylight factor [5, 6]: direct light (from sun), diffuse light (from sky), exterior reflections (from objects outside), and interior reflections (from objects and walls inside). This combination determines the daylight appearance inside buildings.

The light distribution in an interior space is strongly influenced by the type of sky outside. Clear sky types are characterized by a strong directional sun component (3000-5000 K) and a blue diffuse sky component (12000-18000 K). The exact values of intensity and color temperature depend on time of the day, time of the year, and location on earth. Partly clouded skies often show a dynamic pattern in intensity levels, color temperatures, and directionality resulting from moving clouds. The sun behind a cloud results in lower intensity levels, cooler colors, and more diffuse daylight, whereas the daylight appearance of an uncovered sun will resemble more a clear sky situation (directional sun, high intensity levels, and warmer colors). Overcast skies are characterized by diffuse daylight $(6000-7000 \mathrm{~K})$ because the sun is fully covered.

The appearance of the daylight inside a building is strongly connected with the architecture of the facade (for windows) and ceiling (for skylights) layout. The way the sunlight is blocked in the facade or ceiling has a direct influence on the shape and frequency of the patterns in the room. The edge sharpness of the patterns and the color/intensity depend on location on earth, positioning, sky conditions, time of day and year.

Being able to understand the perceptual relation between architectural choices and the attractive qualities of daylight provides valuable insights for maximizing the daylight impression of a room. The aim of this study is to investigate the effect of sky type and skylight configuration on the daylight impression of a room. 


\section{HDR imaging and daylight rendering}

In this research, advanced rendering techniques were used to create realistic daylight impressions inside buildings. The room was build using Google Sketchup and Indigo rendering was used to render the daylight effect in the room. As input for this rendering procedure High Dynamic Range (HDR) image material of real world skies was used.

HDR imaging is a technique that allows a larger dynamic range of luminance between the lightest and darkest areas of an image. Real world scenes with a large intensity range (like daylight 1:100000) can be captured more accurately using 32 bit HDR capturing. The left picture in Figure 1 shows a $360^{\circ}$ HDR image from a partly clouded sky (HyperFocal Design). The right picture shows the $360^{\circ}$ capturing device.
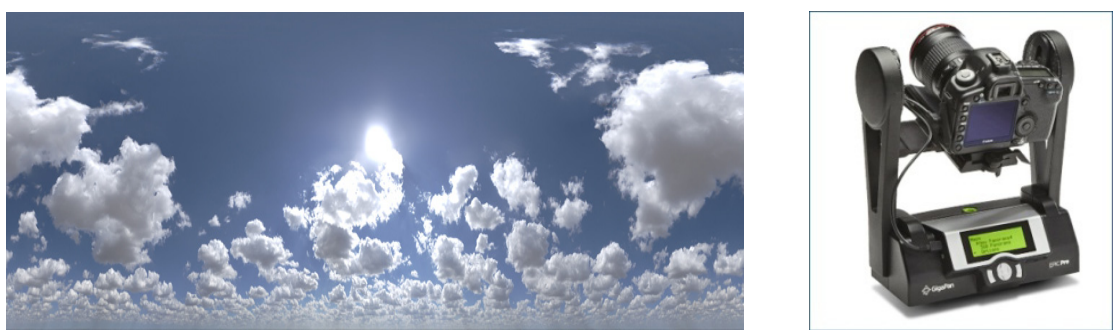

Figure 1: Left panel shows HDR image a partly clouded sky (from HyperFocal Design) and the right panel shows a capturing device.

HDR images are generally achieved by capturing multiple standard photographs from the same view point with different exposure times, and then merging them into an HDR image. The $360^{\circ}$ image is created by making multiple HDR images for different angles. Professional stitching software is used to create one single $360^{\circ} \mathrm{HDR}$ image.

The HDR image in combination with a Sketch-up model of an office is used as input for Indigo rendering software to create the daylight renderings. Section 3.2 describes the stimuli of the experiment in more detail.

\section{Daylight impression experiment}

\subsection{Setup}

The setup consisted of a 42 inch LCD TV panel connected to a PC. The participants were seated in front of the TV at a distance of 1.5 meters. The background illumination on the white wall behind the TV was 20 lux. All other illumination in the room was turned off. 


\subsection{Stimuli}

In Google Sketch-up a room with 'standard' office size $(6 \mathrm{~m} \times 3.5 \mathrm{~m} \times 2.7 \mathrm{~m})$ was created. Figure 2 shows the Sketch-up model.

The walls are plastered (white) and the floor has a gray carpet. The skylight openings in the ceiling have a recess of $10 \mathrm{~cm}$. Indigo renderer was used to render the daylight effects in the room using 4 HDR sky images (clear sky, partly clouded sky sun visible, partly clouded sky sun not visible, and overcast sky). The HDR images were placed around the room as a dome in such a way that the sun position was at the same location for all scenes.

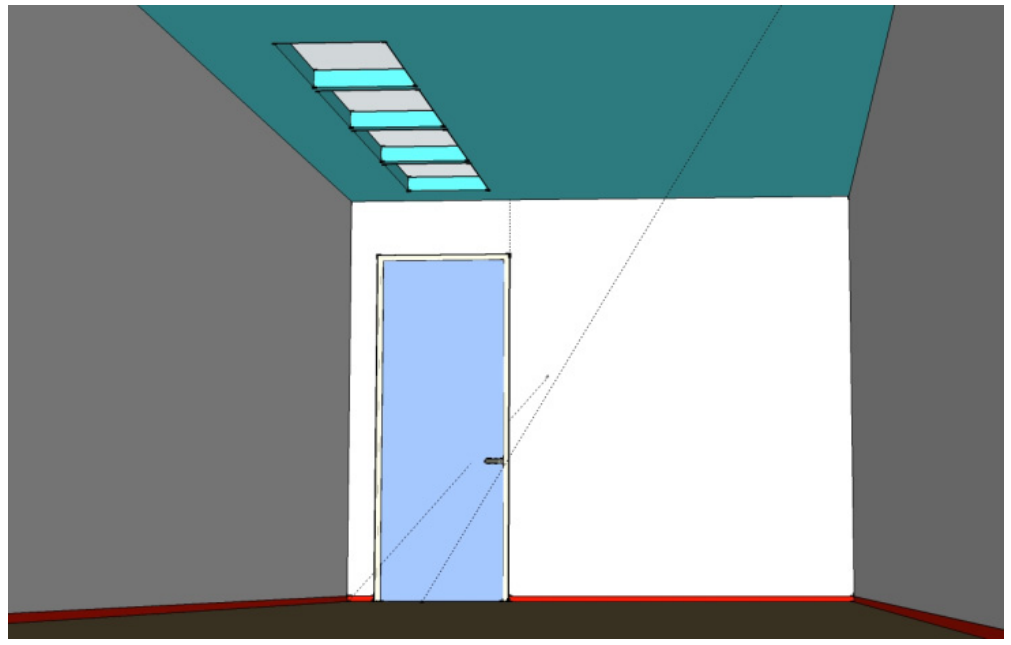

Figure 2: Sketch-up model of the office room.

A perceptually based tone-mapping technique (Reinhard) was used to compress the HDR data into a displayable range in a meaningful manner such that the visual appearance can be reproduced [7].

The stimuli used in this experiment consisted of 5 ceiling configurations and 4 sky types resulting in $20(5 \times 4)$ different renderings. The 5 ceiling configurations are shown in Figure 3.

The 4 sky types (clear sky, partly clouded sky sun visible, partly clouded sky sun not visible, and overcast sky) are shown in Figure 4.

\subsection{Procedure}

Eleven male and nine female observers (all European) participated in the experiment. The participants worked in a research environment but had mixed backgrounds (research, human resources, secretary, students). Their age ranged between 23 and 47 years. All participants had normal color vision. The experiment was conducted using a paired-comparison design, in which two 


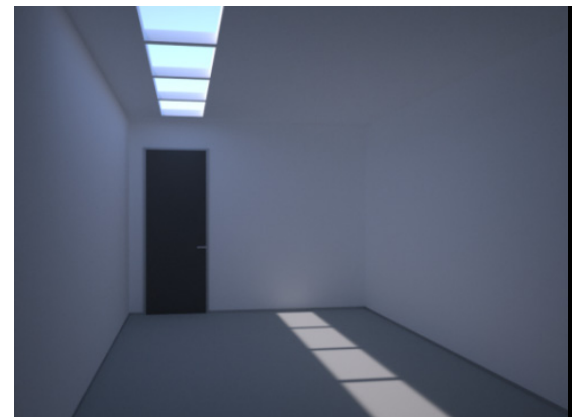

$60 \times 120 \mathrm{~cm}$ right $(4 \mathrm{x})$

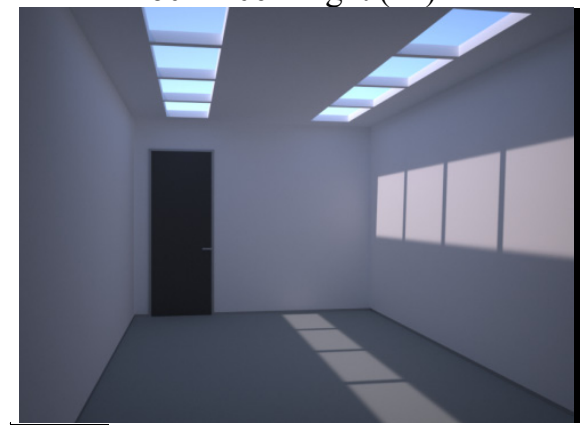

$60 \mathrm{x} 120 \mathrm{~cm}(8 \mathrm{x})$

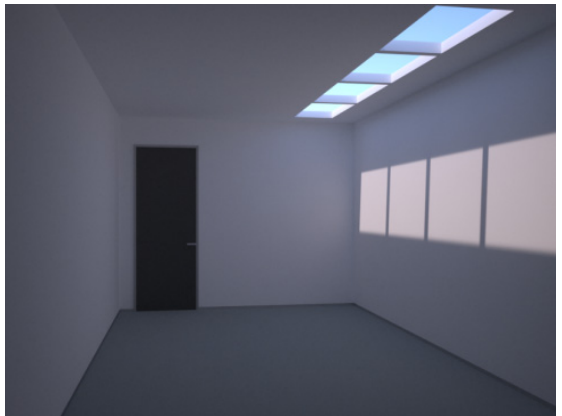

$60 x 120 x m$ left $(4 x)$

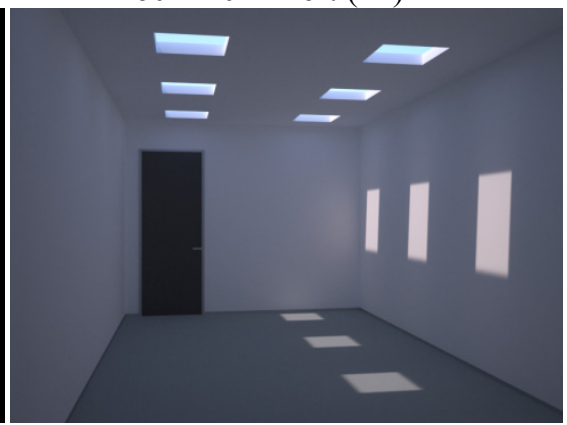

$60 \times 60 \mathrm{~cm}(6 x)$

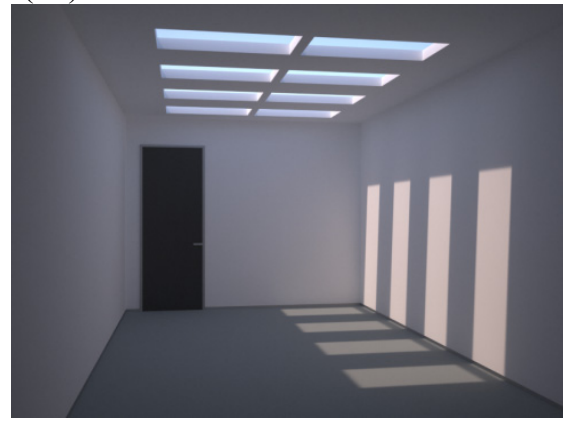

60x120cm (8x) perpendicular

Figure 3: $\quad$ Five ceiling configurations.

images were shown side-by-side. The participants were requested to indicate which image (left or right) had the strongest daylight impression. After each image pair a gray field was shown for 2 seconds.

In the experiment not all possible combinations of the 20 images were shown to the participants but only the comparisons where either 1) sky type was always the same in the left and right image or 2) ceiling configuration was always the same in the left and right image. This setup resulted in 70 side by side comparisons in total. Afterwards in a short interview, the participants were asked to indicate the most important aspects in the image which their judgement was based on. 


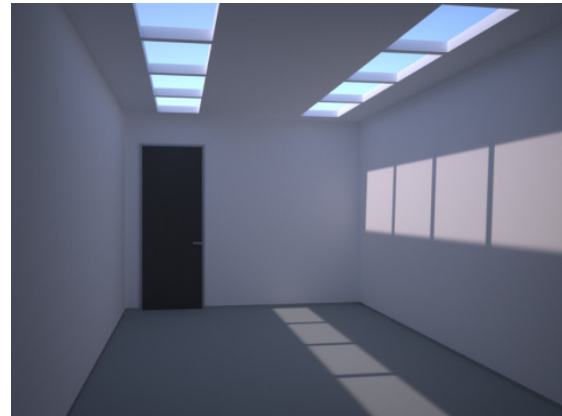

Clear sky

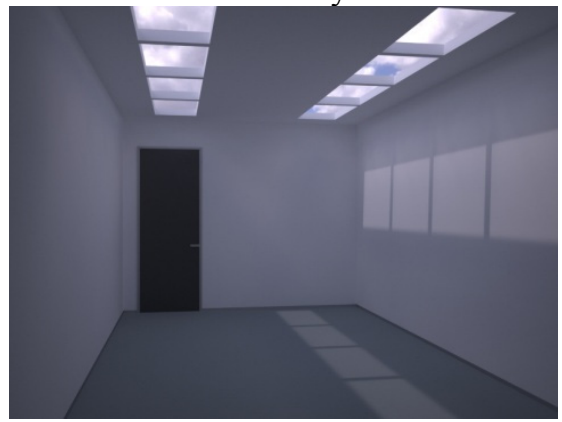

Partly clouded sun visible

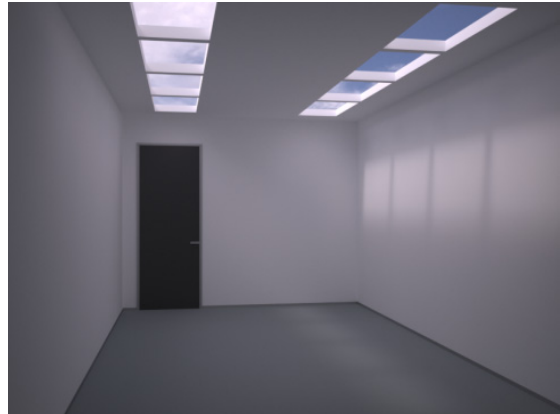

Partly clouded sun behind cloud

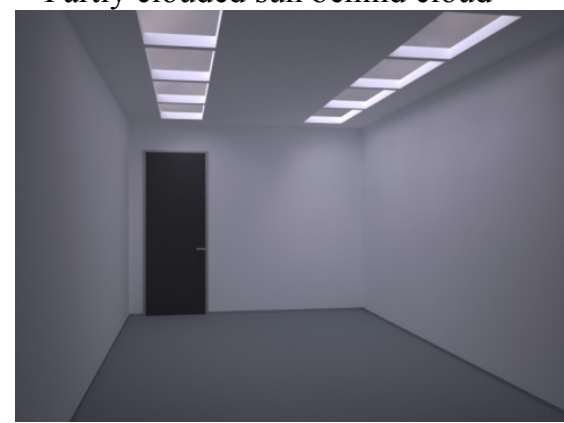

Overcast

Figure 4: $\quad$ Four different sky types.

\section{Results}

Analysis of the main effects was done using Montag graphical model analysis [8]. Figure 5 and 6 show respectively the main effect of sky-type and configuration on people's daylight impression. A $\Delta \mathrm{Z}$ score of $0,0.5,1$, and 1.5 correspond respectively to a percentage of $50 \%, 70 \%, 85 \%$, and $93 \%$ that a condition is preferred above another. The bars around the mean z-scores are modified error bars from Montag's model to make reading of the pair wise significances possible. Results are statistically significant if the mean z-score of one condition is outside the bars of the other level of that variable.

The analysis shows a significant main effect of sky type on the perceived daylight impression of a room (averaged over configurations). Figure 5 shows that the overcast sky scores lowest on daylight impression. Clear sky and the partly cloudy sky with the sun in front of clouds (direct) score highest and do not differ significantly from each other. Approximately $93 \%$ of the people preferred a clear sky and partly clouded sky condition (sun visible) over an overcast sky condition in terms of daylight impression. Approximately $70 \%$ of the people preferred a clear sky and partly clouded sky condition (sun visible) over a partly clouded sky (sun not visible). 


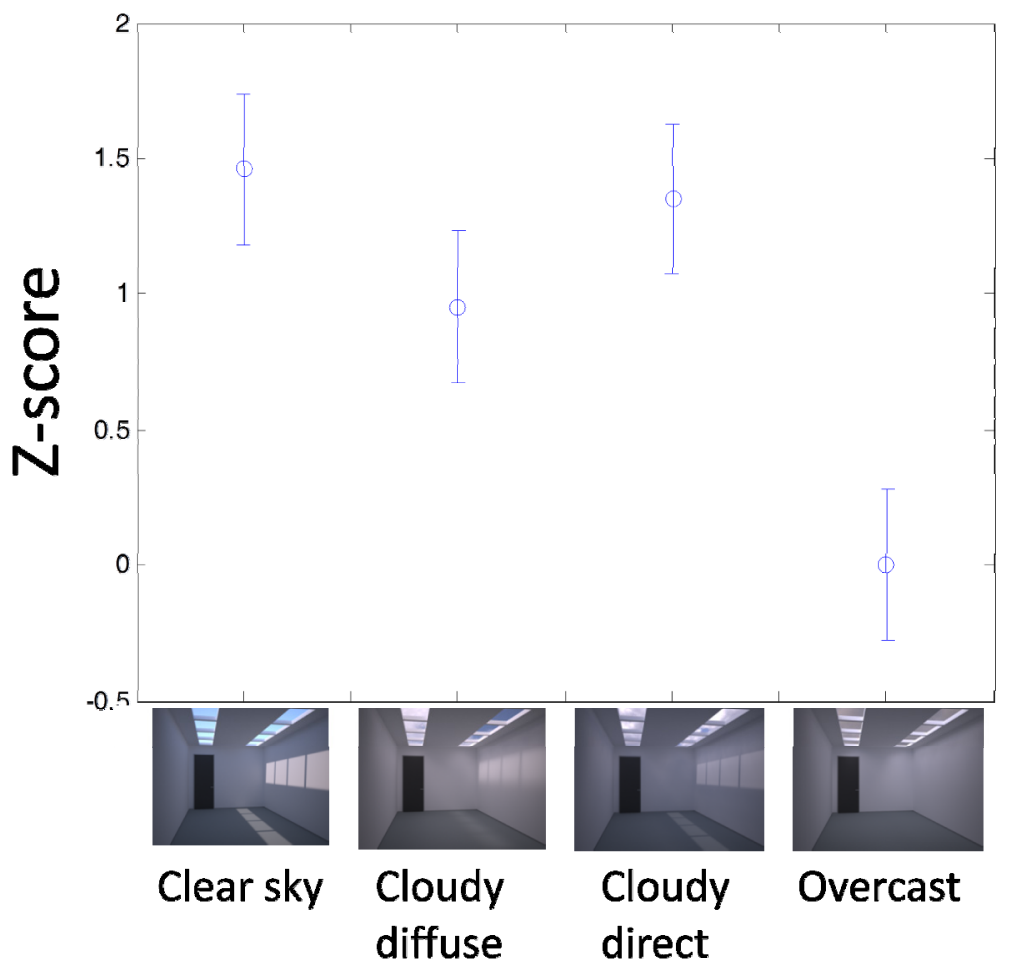

Figure 5: $\quad$ Effect of sky type on the perceived daylight impression. The x-axis shows the four sky types and the y-axis shows the perceived daylight impression (in Z-scores).

The analysis shows a significant main effect of ceiling configuration on the perceived daylight impression of a room (averaged over sky type). Figure 6 shows that configuration 4 with the $60 \times 60 \mathrm{~cm}$ skylights scores significantly lower than the other configurations that have the $120 \times 60 \mathrm{~cm}$ skylights. Configuration 1 and 2 (one sided 4x, 120x60 cm) score significantly lower than the configurations 3 and $5(8 x, 120 \times 60 \mathrm{~cm}$ and perpendicular) on daylight impression. Approximately $90 \%$ of the people preferred configurations 3 and 5 $(8 x, 120 \times 60 \mathrm{~cm})$ over configuration $4(6 x, 60 x 60 \mathrm{~cm})$ in terms of daylight impression and approximately $70 \%$ of the people preferred configuration 1 and 2 (120x60 cm one sided) over configuration $4(6 \mathrm{x}, 60 \times 60 \mathrm{~cm})$.

Interaction effects were analyzed using Monte Carlo parametric bootstrap analysis. No interaction effects were found between ceiling configuration and sky type meaning that the main effect of sky-type holds for all ceiling configurations and vice versa.

The interview results showed that skylight appearance, light pattern, and configuration had the largest impact on the daylight impression. Participants 


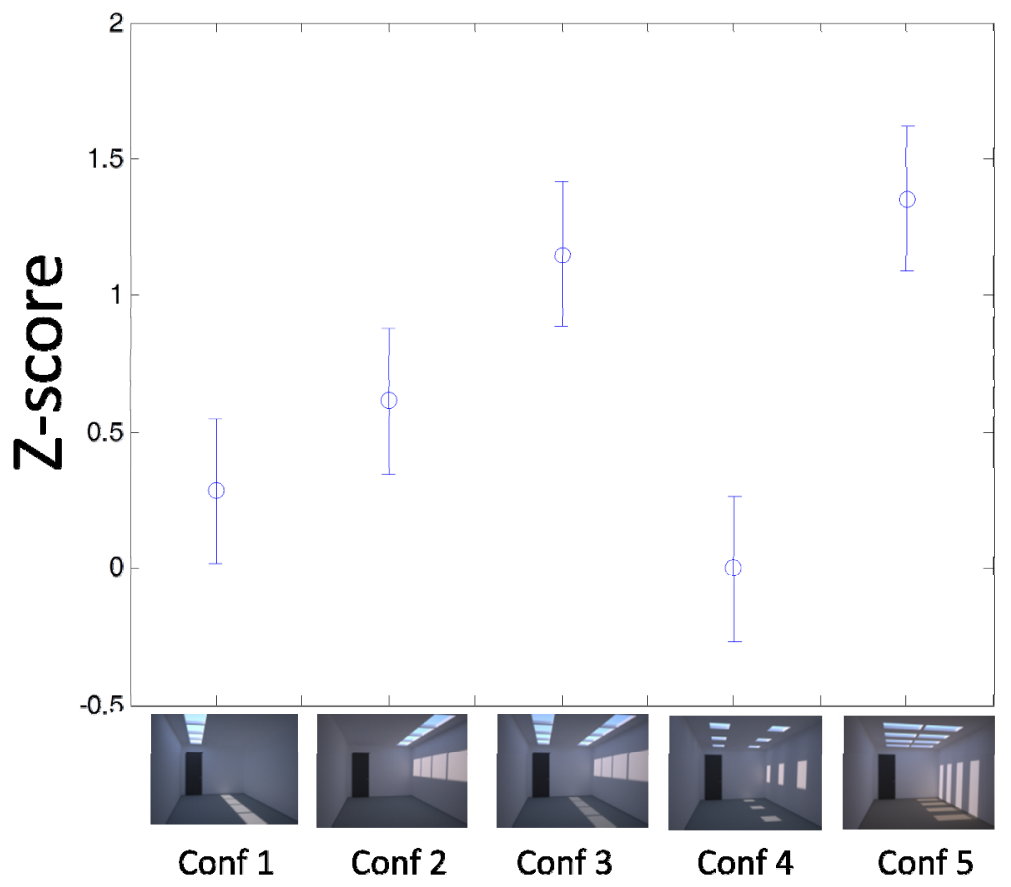

Figure 6: Effect of ceiling layout on the perceived daylight impression. The $\mathrm{x}$-axis shows the five ceiling configurations and the $\mathrm{y}$-axis shows the perceived daylight impression (in z-scores).

preferred large rectangular openings with a blue sky or partly clouded sky. The light patterns in the room should have sharp edges and a warm color temperature for a strong daylight impression. The skylight configuration with rectangular openings $(120 \times 60 \mathrm{~cm})$ on both the left and right side and perpendicular to the viewpoint were preferred. In general people state that the larger the skylight opening the better the daylight impression in the room.

\section{Conclusions}

In general, the results from this perception test appeared to be very robust. The daylight impression of a clear sky and partly clouded sky (sun visible) resulted in the strongest daylight impression. Most people preferred the larger rectangular skylights on both sides of the room or perpendicular to the viewer. In general, there was a strong preference for large rectangular skylights showing blue sky with some clouds, resulting in warm light patterns with sharp edges in room.

The results of this experiment are only based on a selection of sky types and skylight configuration. In future research, different sky types and skylight configurations will be investigated. Furthermore, the effect of dynamics in 
daylight patterns (change in shape, sharpness, color, intensity over time) on the daylight impression will be investigated in the near future.

\section{References}

[1] Boyce, P., C. Hunter, et al. The Benefits of Daylight through Windows. New York, Rensselaer Polytechnic Institute, 2003

[2] Edwards, L. and P. Torcellini. A literature review of the effects of natural light on building occupants. Golden, Colorado, National Renewable Energy Laboratory, 2002

[3] Farley, K. M. J. and J. A. Veitch. A room with a View: A Review of the Effects of Windows on Work and Well-Being. Ottawa, National Research Council Canada (NRC), 2001

[4] Boyce, P. The Impact of Light in Buildings on Human Health. Indoor Built Environment 19(1): 8-20, 2010.

[5] CIE BS EN 15469: Spatial Distribution of Daylight - CIE standard general sky. Vienna, CIE Central Bureau, 2004

[6] Boyce, P. and P. Raynham. The SLL Lighting Handbook. London, The Society of Light and Lighting, 2009.

[7] Reinhard, E., Ward, G., Pattanaik, S. and Debevec, P. High Dynamic Range Imaging, Morgan Kaufmann, Boston, 2006.

[8] Montag, Ethan D. Empirical formula for creating error bars for the method of paired comparison, Journal of Electronic Imaging, vol 15(1), 2006 